\title{
A Study on Breast Feeding Practice of Mothers of Bangladesh: Perspective Dhaka City
}

\author{
Salim Raza ${ }^{1}$, Sabir Hossain ${ }^{2}$, M. Masihul Alam ${ }^{1}$, Abul Kashem Tang ${ }^{1}$, A. T. M. Mijanur Rahman ${ }^{1}$, \\ Asad Ud-Daula ${ }^{1, *}$ \\ ${ }^{1}$ Department of Applied Nutrition and Food Technology, Islamic University, Kushtia, Bangladesh \\ ${ }^{2}$ Department of Biochemistry and Molecular Biology, Jahangirnagar University, Dhaka, Bangladesh \\ Email address: \\ salimrazaiu@yahoo.com(S. Raza), sabiriu@gmail.com(S. Hossain), alam.anft@gmail.com(M. M. Alam), \\ makashemsa@gmail.com(A.K. Tang),mijananftiubd@gmail.com(A. T. M. M. Rahman), asad.uddaula@googlemail.com(A. Ud-Daula) \\ ${ }^{*}$ Corresponding author
}

\section{To cite this article:}

Salim Raza, Sabir Hossain, M. Masihul Alam, Abul Kashem Tang, A. T. M. Mijanur Rahman, Asad Ud-Daula. A Study on Breast Feeding Practice of Mothers of Bangladesh: Perspective Dhaka City. Journal of Food and Nutrition Sciences. Vol. 6, No. 2, 2018 , pp. 63-69. doi: $10.11648 /$ j.jfns.20180602.14

Received: February 5, 2018; Accepted: February 25, 2018; Published: April 23, 2018

\begin{abstract}
Breastfeeding practices of mother are effective way for reducing infant morbidity and mortality. Improper breastfeeding along with inadequate complementary feeding are the main reasons for malnutrition among the children in Bangladesh. Therefore, the aim of this study is to assess and find the breast feeding practice in selected areas of Dhaka city with respect to socio-economic and demographic condition. Here, 250 mothers and their 250 infants were considered to asses using a formative questionnaire. Within this sample size $129(51.6 \%)$ were male infants and 121 (49.4\%) were female infants respectively. This study found that the privilege of breast feeding practice progressively increases with the mother's education that has been started from less than high school level (below 10 classes) to bachelor's level or more than higher. In addition, this study found that about $84.8 \%$ lactating mothers have knowledge on what are colostrums. But surprisingly only $58 \%$ mothers gave colostrums to their infants. Furthermore, we found that the rate of exclusive breast feeding among three months and six months age infants were 51.2\% and 27.2\% respectively. Therefore, according to findings of this study it could be concluded that mothers in the Dhaka city should give more attention on breast feeding to her infants and subsequently prevent infant mortality and get a healthy child.
\end{abstract}

Keywords: Breastfeeding, Socioeconomic-Demographic, Colostrums, Exclusive

\section{Introduction}

Breastfeeding practice of infants is the feeding of babies but exclusive breastfeeding can be defined as a practice whereby the infants receive only breast milk and not even water, other liquids, tea, herbal preparations, or food during the first six months of life, with the exception of vitamins, mineral supplements, or medicines [1]. Exclusive breastfeeding is an ideal nutrition that supports optimal growth and development to infants for approximately first 6 months of age. UNICEF and WHO recommend that children should exclusively breastfeed (no other complementary liquid or solid or plain water) for the first six months of life and those children can be given solid (semi solid) and complementary food after over those period of time. It has been recognized worldwide that breastfeeding is beneficial for both the mother and child, as breast milk is considered the best source of nutrition for an infant [2].

Proper nutrition plays an important role in the physical, mental and emotional development of an infant. Breastfeeding is the only way that gives complete nutrition. Infants and pre-school children are the most vulnerable group among all the groups in our society [3]. UNICEF has pointed out that malnutrition is the biggest and single cause of infant and child mortality. Malnutrition is largely caused by poverty, ignorance, insufficient education and large family size. These factors influence most directly the quality of life and are the true determinants of malnutrition in society. The 
global picture of breast feeding practice is about $86 \%$ for infants $6-11$ months of age in the developing world and for children 12-23 months of age; it is about $37 \%$ in Latin America, in the Caribbean $70 \%$ and $72 \%$ in Africa and Asia [4]. Over two-thirds deaths occurring worldwide during the first year of life children are often associated with inappropriate feeding practices, especially due to poor exclusive breastfeeding practices [5]. However, rates of malnutrition in Bangladesh are among the highest in the world, with six million children estimated to be chronically undernourished [6], where only 14 per cent of infants were exclusively breastfed up to 3 months were found in Bangladesh [7]. In addition, the proportion of Bangladeshi women initiating breast-feeding is relatively low [8-10]. It may be caused by low levels of awareness of mothers regarding appropriate breast-feeding practices in Bangladesh [11-13]. Furthermore, in recent year there are no data available on breastfeeding practice among Bangladeshi urban women. That's why this study aims to collect recent data of breastfeeding practice among Bangladeshi mothers. This present study determined infant feeding practice and its predictors among mothers in Dhaka City: (1) to assess breastfeeding practices in terms of initiation, exclusivity and termination. (2) to collect baseline data before and at the end of programmes aimed at improving nutrition. (3) to increase the commitment of governments, international organization and other concerned parties for optimal feeding practices.

\section{Methodology}

\subsection{Study Subject}

The present study was conducted in selected area of Dhaka city: Motijheel, Ramna, Dhanmondi and Lalbag during the period of June-December, 2015. A total 250 children's and 250 mothers were selected from the study area. However, the mothers were selected randomly and they are not poor or rich, mostly they have average income.

\subsection{Study Procedure}

The mothers were asked face to face interview by structured questionnaire. A survey teams containing three (03) members, who were assigned to visit the mothers. It was also assigned that the survey team will not visit more than ten mothers per day to ensure quality of data to be collected and subsequent minimization of the bias of the survey. Every day, the quality of interview and collected data were discussed within team member to ensure data competency. In case of any incomplete and inconsistent data, the team were revisited the relevant respondent mothers. Additionally, an informed written consent was taken from every respondent explaining the objectives of this study and it was confirmed that the personal information will be confidential preserved and they could any time withdraw their opinion. In addition, this study was conducted according to the guidelines laid down in the Declaration of Helsinki protocols.

\subsection{Demographic and Socio-Economic Characteristics}

Demographic and socio-economic characteristics were collected by designed questionnaire. Age, sex and birth order of children were recorded. The age of breastfeeding mother can classified into below $<20$ age group, (21-25) age group, (26-30) age group and above 30 age group. Education level of breastfeeding mothers were categories into less than high school, high school, college or associates and bachelors or higher level. Occupation of mothers was also classified into unemployee, govt. employee, and private employee. Family income can be categories into low income family and high income family based on mother poverty income ratio (PIR). The religion practice of mothers also recorded.

\subsection{Analysis of Data}

Data were analyzed using the SPSS software (version 11.5). Frequencies were calculated for descriptive analysis and chi-square tests were used to evaluate relationship between different selected variables.

\section{Results}

\subsection{Study of Children Based on Sex, Age and Birth Order}

In this study, 250 children's in Dhaka City were classified on the basis of their sex, age and birth order. Here higher number of male children, $129(51.6 \%)$ and little lower number of female children $121(48.4 \%)$ were found in the study area. In addition, children also sorted out on the basis of age group. Most of the children were selected in the age group below 1 year and (1-2) years which is about $90(36 \%)$ and $105(42 \%)$ respectively. The remaining $40 \quad(16 \%)$ children's were found who have age between (2-3) years, and 15 (6\%) children's were found who have age between (3-5) years. Furthermore, Here, 85 (34\%) children, $110(44 \%)$ children, $55(22 \%)$ children were found for first born, second born and above born two respectively.

\subsection{Religion Practice and Age Group Study of Lactating Mother in the Study Area}

Here, lactating mother were assessed based on religion practice and age group. 205 (82\%) mothers, 35 (14\%) mothers, and $10(4 \%)$ mothers were found to be muslin, Hindu and other religion respectively. In addition, 58 (23.2\%) lactating mothers were found who have age below 20 years, 88 (35.2\%) lactating mothers were found who have age between (21-25) years, 66 (26.4\%) lactating mothers were found who have age between (26-30) years, and 38 $(15.2 \%)$ mothers were found who have age above 30 years.

\subsection{Socio-Demographic Characteristics of the Study Subjects}

This study found that $60(24 \%), 77(30.8 \%), 80(32 \%)$ and $33(13.2 \%)$ mothers were educated as for less than high school level education, high school or equivalent education level, college or associates degree level and bachelors degree 
or higher education level respectively. Furthermore, 140 (56\%), 63 (25.2\%), and 47 (18.8\%) mothers were found to be unemployed (house wife), Govt. employed and private employed respectively. Also, this study found 167 (66.8\%) mothers were in low income and $83(33.2 \%)$ mothers were found in high income. All the numerical data were presented in the Table 1 .

Table 1. Educational level, occupation and Poverty Income Ratio (PIR) of lactating mother in the Study Area.

\begin{tabular}{lll}
\hline Characteristics & Number \& Percentage & Chi square value \\
\hline Mothers education & $60(24 \%)$ & \\
Less than high school & $77(30.8 \%)$ & \\
High school & $80(32 \%)$ & 0.856 \\
College or associates degree & $33(13.2 \%)$ & \\
Bachelors or higher & $140(56 \%)$ & \\
Mothers occupation & $63(25.2 \%)$ & \\
Un-employee & $47(18.8 \%)$ & \\
Govt. employee & $167(66.8 \%)$ & 1.92 \\
Private employee & $83(33.2 \%)$ & \\
PIR of Mothers & & \\
Low income & & \\
High income & & \\
\hline
\end{tabular}

\subsection{Knowledge and Practice of Lactating Mothers on Colostrums}

In this study, 250 lactating mothers were assessed on the basis of knowledge and practice of colostrums. We found here, most of the lactating mother $(84.8 \%)$ has knowledge what are colostrums. However, surprisingly only $58 \%$ lactating mother who were provided colostrums to their baby.
Data were presented in the Table-2.

Table 2. Knowledge and practice of lactating mothers on colostrums.

\begin{tabular}{lll}
\hline & Yes (\%) & No (\%) \\
\hline Knowledge on colostrums & 84.8 & 15.2 \\
Practice on colostrums & 58 & 42 \\
\hline
\end{tabular}

\subsection{The Study of Lactating Mothers Based on Infant Feeding Practice}

Table 3. Distribution of study population according to practice of infant feeding.

\begin{tabular}{|c|c|c|c|}
\hline Mother's infant feeding practice & & Number (n) & Percentage $(\%)$ \\
\hline \multirow[t]{4}{*}{ First food of new born } & Colostrums & 145 & $(58 \%)$ \\
\hline & Honey & 35 & $(14 \%)$ \\
\hline & Water & 42 & $(16.8 \%)$ \\
\hline & Others & 28 & $(11.2 \%)$ \\
\hline \multirow[t]{2}{*}{ Breast feeding initiation after birth } & Within 1-3 hours & 170 & $68 \%$ \\
\hline & First day & 50 & $20 \%$ \\
\hline \multirow[t]{2}{*}{ Exclusive breastfeeding up to 3 month } & Yes & 128 & $51.2 \%$ \\
\hline & No & 122 & $48.8 \%$ \\
\hline \multirow[t]{2}{*}{ Exclusive breastfeeding up to 6 month } & Yes & 68 & $27.2 \%$ \\
\hline & No & 182 & $72.8 \%$ \\
\hline
\end{tabular}

In this study, 250 lactating mothers were studied on the basis of providing first baby food at birth. Where, 145 (58\%) mothers stated that the baby's first food should be colostrums followed by water $42(16.8 \%)$, honey $35(14 \%)$ and other 28 (11.2\%). In addition, study population also sorted out according to the breastfeeding initiation after birth. Where, only $170(68 \%)$ mothers assumed that breastfeeding should be initiated within 1-3 hours, $50(20 \%)$ mothers thought this should be within 1 day and $30(12 \%)$ stated 2 day or later. Furthermore, 250 children were assessed based on the duration of exclusive breast-feeding. Where only $51 \%$ baby's were found to continuing exclusive breast-feeding at 3 months of period and $27 \%$ baby's were found to continuing exclusive breast-feeding at 6 months of period. All the numerical Data were presented in the Table-3.

\subsection{Breast-Feeding Prevalence of Infants Until 6 Months Depending on Various Study Variables}

There are many variables such as sex, maternal age, birth order, educational level, employment status and poverty income ratio were considered to find the breast-feeding prevalence of infants until six (06) months. 250 children from the study area were assessed in this study. In considering sex variable, there were $76(58.91 \%)$ male children and 72 $(59.50 \%)$ female children were found out of 250 target children who continued breast-feeding until six months of period as presented in the Table- 4 . However, considering maternal age about $30(51.72 \%)$ mothers were found for age 
less than 20 years, $51(57.95 \%)$ mothers were found for age between $(21-25)$ years, $41(62.12 \%)$ mothers were found for age between (26-30) years and $22(57.89 \%)$ mothers were found for age above 30 years out of 250 mothers who continued breast-feeding until 6 months age of their infant as illustrated in the Figure-1.

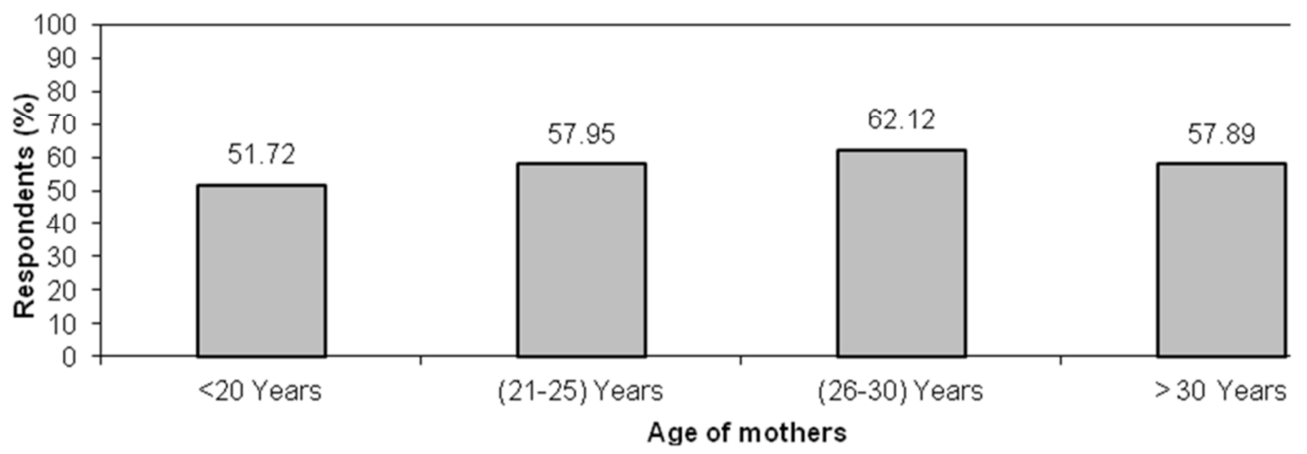

Figure 1. Breast-feeding given to infants at 6 months in Dhaka City depending on maternal age.

Additionally, in this study 250 children were selected from Dhaka depending on the birth order of children. Where 53 (62.35\%) children, $64(58.18 \%)$ and $29(52.72 \%)$ children were found for first born, second born and above born two respectively out of 250 children who continued breast-feeding until six (06) months of period. The data were illustrated in the Figure 2.

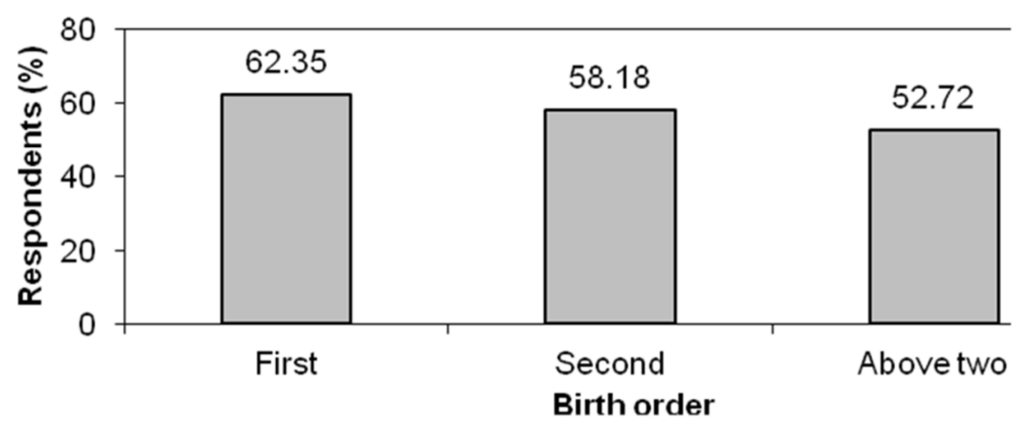

Figure 2. Percentage of breast-feeding until 6 months of period depending on birth order of child.

Furthermore, 250 lactating mothers were selected in this study based on maternal educational level. Where $33(55 \%)$ mothers, 44 (57.14\%) mothers, 49 (61.25\%) mothers and 22 $(66.66 \%)$ mothers were found for less than high school level education, high school or equivalent education level, college or associates degree level and bachelors degree or higher education level respectively out of 250 mothers who continued breast-feeding until 6 months age of their infant as illustrated in the Figure 3.

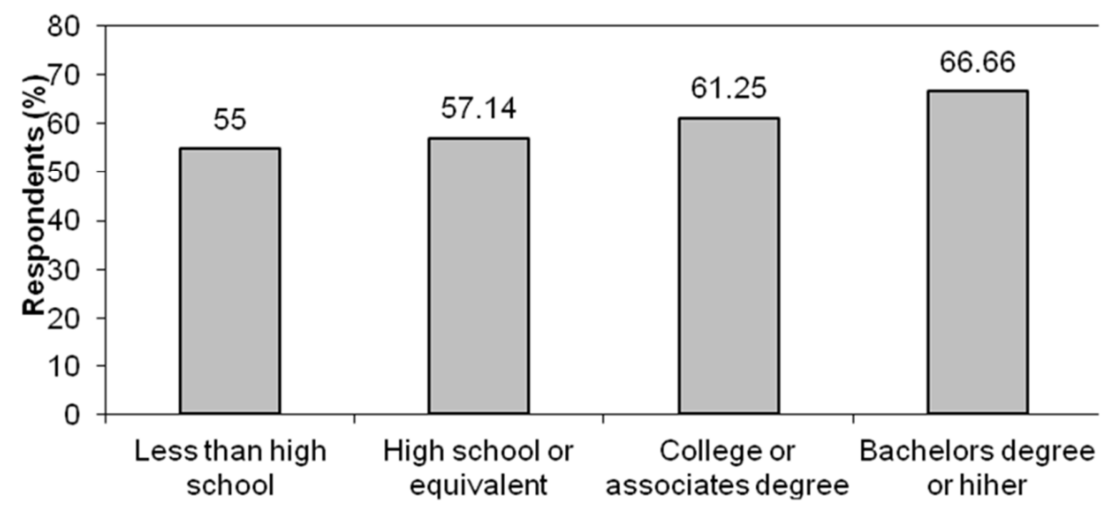

Figure 3. Percentage of breast-feeding until 6 months of period depending on maternal educational level in Dhaka City.

Additionally, in this study lactating mothers were selected based on maternal employment status. Where $91(65 \%)$ mothers, 34 (53.96\%) mothers, and 20 (42.55\%) mothers were found for unemployed (house wife), Govt. employed and private employed respectively out of 250 mothers who continued breast-feeding until 6 months age of their infant.
Furthermore, on the basis of Poverty Income Ratio (PIR), where $96(57.48 \%)$ mothers were found in low income and $50(60.24 \%)$ mothers were found in high income out of 250 mothers who continued breast-feeding until 6 months age of their infants as presented in the Table- 4 . 
Table 4. Prevalence of breastfeeding by selected characteristics of mothers.

\begin{tabular}{lll}
\hline Characteristics & Breastfeeding at 6 months & Breastfeeding at 12 months \\
\hline & $\mathbf{( \% )}$ & (\%) \\
\hline Sex of child & $58.91 \%$ & $33.33 \%$ \\
Male & $59.50 \%$ & $35.53 \%$ \\
Female & & \\
Mothers age (Year) & $51.72 \%$ & $29.31 \%$ \\
$<20$ & $57.95 \%$ & $34.09 \%$ \\
$(21-25)$ & $62.12 \%$ & $37.87 \%$ \\
$(26-30)$ & $57.89 \%$ & $34.21 \%$ \\
$>30$ & & \\
Birth order of child & $62.35 \%$ & $36.47 \%$ \\
First & $58.18 \%$ & $34.54 \%$ \\
Second & $52.72 \%$ & $30.90 \%$ \\
Above two & & \\
Mothers education & $55 \%$ & $36.66 \%$ \\
Less than high school & $57.14 \%$ & $37.66 \%$ \\
High school & $61.25 \%$ & $41.25 \%$ \\
College or associates degree & $56.66 \%$ & $38.42 \%$ \\
Bachelors degree or higher & & \\
Mothers occupation & $65 \%$ & $36.42 \%$ \\
Unemployee & $53.96 \%$ & $31.74 \%$ \\
Govt. employee & $42.55 \%$ & $27.65 \%$ \\
Private employee & $57.48 \%$ & $34.73 \%$ \\
Mothers PIR & $60.24 \%$ & $32.53 \%$ \\
Low income & &
\end{tabular}

\subsection{Breast-Feeding Prevalence of Infants until 12 Months Depending on Various Study Variables}

In this study, there were many variables such as sex, maternal age, birth order, educational level, employment status and poverty income ratio which considered to find the breast-feeding prevalence of infants until twelve (12) months. On the basis of sex variable, there were about $43(33.33 \%)$ male children and 43 (35.53\%) female children were found out of 250 target children who continued breast-feeding until twelve (12) months of period. However, considering maternal age, here, $17(29.31 \%)$ mothers were found for age less than 20 years, $30(34.09 \%)$ mothers were found for age between (21-25) years, 25 (37.87\%) mothers were found for age between (26-30) years and $13(34.21 \%)$ mothers were found for age above 30 years out of 250 mothers who continued breast-feeding until twelve (12) months age of their infant. Additionally, in this study 250 children were selected from the study area depending on the birth order of children. Where 31 (36.47\%) children, 38 (34.54\%) and 17 (30.90\%) children were found for first born, second born and above born two respectively out of 250 children who continued breast-feeding until twelve (12) months of period. Furthermore, based on maternal educational level, where 22 (36.66\%) mothers, 29 (37.66\%) mothers, 33 (41.25\%) mothers and $14(42.42 \%)$ mothers were found for less than high school level education, high school or equivalent education level, college or associates degree level and bachelors degree or higher education level respectively out of 250 mothers who continued breast-feeding until twelve (12) months age of their infant. Furthermore, 250 children were classified on the basis of Poverty Income Ratio (PIR) from the study area. Where $58(34.73 \%)$ mothers were found in low income and $27(32.53 \%)$ mothers were found in high income out of 250 mothers who continued breast-feeding until twelve (12) months age of their infant. All the numerical data were presented in the Table- 4 .

\section{Discussion}

The community based cross sectional study which was conducted on 250 mothers and their 250 children in the Dhaka City. The target respondents were selected randomly and assed mother breast-feeding practice. A picture was found between target populations of that selected areas in respect to socio-economic and demographic condition. The birth frequency of male children was found higher in this study area and this result has been supported the previous literature which claims that in the world population where total male was higher than female [14]. Furthermore, this study was found a significant number of children who have age between (1-2) years and the lowest numbers of children were found who have age between (3-5) years in the study area as presented in the Table-1. However, in this study the un-employment mother was significantly more due to lack of work facilities in the study area.

The breast feeding practices of the mother in this study were assessed based on knowledge and practice of colostrums, first baby food feeding, initiation of breast feeding and duration of exclusive breastfeeding. Considering the knowledge and practice of colostrums, where $84.8 \%$ lactating mothers who have knowledge what are colostrums but only 58\% mothers giving colostrums on their children in Dhaka City. A similar study was conducted in Matlab, 
Bangladesh, reported that only 60 percent of the infants were given colostrums $[15,16]$.

In the Dhaka City, 51\% children's were found to continuing exclusive breast-feeding at 3 months of period and $27 \%$ children's were found to continuing exclusive breast-feeding at 6 months of period that indicates that lactating mother in Dhaka city are reluctant to exclusive breast feeding. The breast feeding prevalence of infants until six (6) and twelve (12) month of period was determined respectively according to sex of child, maternal age, birth order, employment status of mother and poverty income ratio of mother. There was no discrimination detected for either male or female children in the Dhaka city on the privilege of breast feeding offered by mother until six and twelve month age of infant. Additionally, about $37.87 \%$ mothers at age of (26-30) years continue their breast feeding until 12 month of infant age as well mother age above 30 years was found almost similar frequency of her breast-feeding to infants. The previous literatures were also strengthened $[17,18]$ the breast feeding findings was gradually decreased with the ascending birth order of child. The first baby was received more privilege of breast feeding than second baby and it was consequent for above two baby. Grummer et.al reveals that in developing countries, higher education was found to be associated with shorter duration of breastfeeding [19]. However, this study found the breast feeding privilege gradually increases with the mother education started from less than high school level to college or associated level except for Bachelors degree or higher as well significant higher breast feeding privilege was found for the unemployment mother in the study area may be due to the available time in her hands. And a contrary result found for government and private employee mother.

\section{Conclusion}

The study revealed breastfeeding practice of mothers in Dhaka City from the specific point of view of socioeconomic and demographic situation. The infant's growth and development mainly depends on the maternal status, number of children, family income and educational status. Some factors specially sex, maternal age, birth order, educational level, employment status and poverty income ratio of mother that influence children feeding practices until 6 months of period and until 12 months of period. The findings of the present study stress on empowering women with education and economic independence that can help to achieving a healthy environment for mother and children.

\section{Acknowledgements}

The authors wish to thank the study respondent's mothers who gave their valuable time and contribute in this research. Additionally, authors wish to confirm that there are no known conflicts of interest associated with this publication and there has been no significant financial support for this work that could have influenced its outcome.

\section{References}

[1] T. E. Nkala and S. E. Msuya, "Prevalence and predictors of exclusive breastfeeding among women in Kigoma region, Western Tanzania: a community based cross-sectional study," International Breastfeeding Journal, Vol. 6, No. 17, 2011.

[2] C. Ku and S. K. Y. Chow, "Factors influencing the practice of exclusive breastfeeding among Hong Kong Chinese women: a questionnaire survey," Journal of Clinical Nursing, Vol. 19, No. 17, pp. 2434-2445, 2010.

[3] Park K. Park's Textbook of Preventive and Social Medicine. 18th ed. Publishers: Banarsidas Bhanot, Jabalpur, India. Jan. 2005: $391-399$.

[4] Lauer, J. A., Betran, A. P., Victora, C. G., de Onis, M. \& Barros, A. J. D. "Breastfeeding Patterns and Exposure to Suboptimal Breastfeeding among Children in Developing Countries: Review and Analysis of Nationally Representative Surveys," BMC Medicine, Vol. 2, No. 26, 2004.

[5] WHO. Exclusive Breastfeeding for Six Months Best for Babies. Geneva: WHO; 2011.

[6] DHS, 2011. UN population project, Save the Children calculation. Estimated for 2011.

[7] National Institute of Population Research and Training (NIPORT), Bangladesh Demographic and Health Survey 1996-97. Calverton, Maryland: NIPORT, Mitra and Associates and Macro International; pp. 129-33; 1997.

[8] Ahmed S, Parveen SD, Islam A. Infant feeding practices in rural Bangladesh: policy implication. J Trop Paediatr; Vol. 45, pp. 37-41, 1999.

[9] Bangladesh Breastfeeding Foundation (BBF). Situation of breastfeeding in Bangladesh. BBF Research Bull; Vol. 7, No. 2, pp. 4-5, 2001.

[10] Haider R, Kabir I, Ashworth A. Are breastfeeding promotion messages influencing mothers in Bangladesh? Results from an urban survey in Dhaka, Bangladesh. J Trop Pediatr; Vol. 45, pp. 315-8; 1999.

[11] Zeitlyn S, Rowshan R. Privileged knowledge and mothers' "perceptions": the case of breast-feeding and insufficient milk in Bangladesh. Med Anthropol Q; Vol. 11, pp. 56-68, 1997.

[12] Das DK, Ahmed S. Knowledge and attitude of the Bangladeshi rural mothers regarding breastfeeding and weaning. Indian J Pediatr; Vol. 62, pp. 213-7, 1995.

[13] Dewey KG, Adu-Afarwuah S. Systematic review of the efficacy and effectiveness of complementary feeding interventions in developing countries. Maternal \& Child Nutrition. 4 (Suppl 1):24-85, 2008.

[14] Population by sex for the United States, Regions and States and Puerto Rico: www.census.gov/prod/doc/sf1.pdf/ cen2000.

[15] International Centre for Diarrhoeal Disease Research, Bangladesh. Demographic surveillance system; early indicators, Matlab-1993. Dhaka: International Centre for Diarrhoeal Disease Research, Bangladesh, 1994.

[16] Shameem Ahmed, Ariful Islam, Sadia D Parveen. Infant Feeding Practice in Rural Bangladesh: Policy implications. ICDDR, B: Centre for Health and Population Research. [WP 108, 1998]. 
[17] Heath AL, Tuttle CR, Simons MS, Cleghorn CL, Parnell WR. A longitudinal study of breast-feeding and weaning practices during the first year of life in Dunedin, New Zealand. J Am Diet Assoc. 102 (7): 937-43. Available PMID: 12146555; July 2002.

[18] Margaret M. McDowell, M. P. H., R. D.; Chia-Yih Wang, Ph. D.; and Jocelyn Kennedy-Stephenson, M. S. Breastfeeding in the United States: Findings from the National Health and
Nutrition Examination Surveys, 1999-2006. NCHS Data Brief. No. 5, April 2008.

[19] Grummer-Strawn LM. The effect of changes in population characteristics on breast-feeding trends in fifteen developing countries. Int J Epidemiol; Vol. 25, pp. 94-102, 1996. 